$63^{\text {ème }}$ Congrès de la SFCO, 02034 (2015)

DOI:10.1051/sfco/20156302034

(C) Owned by the authors, published by EDP Sciences, 2015

\title{
COMMUNICATION
}

\section{A calcified onchocercal nodule in the infratemporal fossa. An uncommon case}

\author{
Mauprivez C, Harlay P, Gruffaz F \\ Conf sfco, Groupe Hospitalier, Pitié Salpétrière 47-83 boulevard de l'Hopital, 75651 Paris
}

Onchocerciasis, or river blindness, is a parasitic infection caused by the filarial nematode, Onchocerca volvulus. The parasite infects at least 25 million people worldwide. Onchocerciasis is currently endemic in tropical Africa, Latin America and Yemen. The infection is transmitted to humans by blackflies of the genus Simulium that transfer infective larvae during a blood meal. In subcutaneous tissues the larvae develop into adult filariae, which reside in fibrous nodules known as onchocercomas. Adult worms can live in the nodules for approximately 15 years. A fertilised female produces millions of microfilariae during her lifetime. Older nodules often contain necrotic material with calcified remnants of filariae. Classical manifestations include itchy skin rashes, subscutaneous nodules and sclerosing keratitis (1). We describe the first case of calcified onchocercoma localised in the infratemporal fossa.

A 33-year-old African woman presented with a painless mass in the right vestibular maxillary. The oral mucosa appeared to be normal, and no surface colour variation was observed. The lesion was well defined, firm, mobile, and slightly tender on palpation. There was no evidence of cervical lymph node enlargement, and a clinical examination of the head and neck showed no abnormalities. An orthopantomogram revealed a radiopaque heterogeneous ovoid lesion, with clear limits, from the maxillary molar apex to the lower fissure of the orbit. Many bone density elements that evoke calcifications were present in the lesion. Axial Computed Tomography imaging with the soft-tissue window setting showed that the lesion was localised inside the buccal fat pad in the infratemporal fossa. Magnetic resonance imaging showed a well-defined lesion, with low signal intensity on T1 and T2-weighted images, evoking first benign calcinosis tumours or calcific phlebolit. The lesion was removed by an intra-oral excision under local anesthesia. The histological examination revealed a calcified onchocercal nodule. Following the pathology results, the patient was referred for a medical evaluation. No other nodule was found on palpation. A skin snip and ophthalmological examination (with a slit lamp) were performed to eliminate systemiconchocerciasis as the diagnosis. There was no finding of microfilaria, and no chemotherapy was necessary.

Numerous parasitoses such as cysticercosis, hydatid cysts or echinococcosis, trichinellosis, dracunculiasis and onchocerciasis might evolve into calcified tumours or dystrophic calcifications. Larvae that are alive are not visible radiographically. Parasite death and calcification development in subcutaneous and muscular sites allow the identification of larvae by X-ray imaging. Many oral cysticercosis cases in the masticatory muscles, tongue, oral mucosa and lips are reported; a calcified cysticercosis nodule in the cheek was described in one case. Three cases of onchocerciasis of the face and mouth and one case of an onchocercoma inside the temporalis muscle have been reported in the literature $(2,3)$. No calcified onchocercal nodule case in the oral and maxillofacial regions has been described. Calcified nodules are typically observed in the scrotal region and around the hip and elbow. ${ }^{3}$ 
An onchocercoma should be included in the differential diagnosis of intra oral nodules or calcinosis tumours in the maxillofacial region in African populations or in expatriate individuals from endemic areas.

1. Simonsen PE, Fischer PU, Hoerauf A, Weil GJ. The filariases. In: Farrar J, Hoter P, Junghanss T, Lalloo D, White NJ, eds. Manson's tropical diseases. $23^{\text {rd }}$ ed. London: WB Saunders Compagny, Ltd., 2013. 737-65.

2. Williams HK, Edwards MB, Adekeye EO. Onchocerciasis of the face and mouth. Oral Surg Oral Med Oral Pathol 1986; 62: 560-3.

3. Martinez RM, Saracho AO. Intramucularonchocercoma. Case report. J Craniomaxillofac Surg 1992; 20: 261-3.

Cédric MAUPRIVEZ

mauprivez.cedric@gmail.com 